October 2017, Volume 2, Issue 4 (80-88)

\title{
Updated Meta-Analysis of Randomized Trials Comparing Safety and Efficacy of Intraoperative Defibrillation Testing with No Defibrillation Testing On Implantable Cardioverter-Defibrillator Implantation
}

\author{
Carlo Bonanno ${ }^{1,}$, Antonio Rossillo ${ }^{1}$, Mariemma Paccanaro ${ }^{1}$,
} Angelo Bruno Ramondo ${ }^{1}$, Antonio Raviele ${ }^{2}$

${ }^{1}$ MD, Department of Cardiology, San Bortolo Hospital, Viale Rodolfi 37, 36100

Vicenza, Italy

${ }^{2}$ MD, FESC, Alliance to Fight Atrial Fibrillation, Via Torino 151/c, 30174 MestreVenice, Italy

* Corresponding author: Carlo Bonanno, MD, Department of Cardiology, San Bortolo Hospital, Viale Rodolfi 37, 36100 Vicenza, Italy. E-mail: carlo.bonan-

DOI: $10.21859 /$ ijcp-020405 no@8aulss.veneto.it

Submited: 04.09.2017

Accepted: 09.22.2017

Keywords:

Defibrillation Testing

Defibrillators, Implantable

Meta-Analysis

(c) 2017. International Journal of Cardiovascular Practice.

\begin{abstract}
Introduction: There is an ongoing debate regarding the need to conduct intraoperative defibrillation testing (DFT) at the time of implantable cardioverter-defibrillator (ICD) implantation. To provide sufficiently strong evidence for the feasibility of omitting intraoperative DFT in clinical practice, we conducted a meta-analysis of randomized controlled trials (RCT) comparing patients with DFT and no-DFT.

Methods: We systematically searched Medline (via PubMed), ClinicalTrial.gov, the Cochrane Central Register of Controlled Trials, and Embase for studies evaluating DFT vs. no-DFT on ICD implantation with regard to total mortality and arrhythmic death, efficacy of first and any appropriate shock in interrupting ventricular tachycardia (VT)/ ventricular fibrillation (VF), and procedural adverse events. Effect estimates [risk ratio (RR) with 95\% confidence intervals (CI)] were pooled using the random-effects model. Results: Our meta-analysis included 4 RCTs comprising 3770 patients (1896 with DFT and 1874 without DFT $)$. Total mortality $(\mathrm{RR}=1.00,95 \%$ CI $0.86-1.17 ; \mathrm{P}=0.98)$ and arrhythmic death $(\mathrm{RR}=1.60,95 \% \mathrm{CI} 0.46-5.59: \mathrm{P}=0.46)$ were not statistically different. Both first $(\mathrm{RR}=0.94,95 \% \mathrm{CI} 0.89-0.98 ; \mathrm{P}=0.004)$ and any appropriate ICD shock $(R R=0.97,95 \%$ CI $0.95-1.00 ; \mathrm{P}=0.02)$ significantly increased the rate of $\mathrm{VT} / \mathrm{VF}$ interruption in the group with no-DFT in comparison with DFT. Finally, the incidence of adverse events was lower in no-DFT patients $(\mathrm{RR}=1.23$; 95\% CI 1.00 $1.51 ; \mathrm{P}=0.05)$.

Conclusions: The practice of DFT (as opposed to no-DFT) did not yield benefits in mortality or the overall rate of conversion of VT/VT. Moreover, a slightly higher incidence of perioperative adverse events was observed in the DFT group.
\end{abstract}

\section{INTRODUCTION}

Testing of the implantable cardioverter-defibrillator (ICD) for its ability to correctly sense, detect and terminate ventricular fibrillation (VF) has been an important part of device implantation since procedures in humans began in the early 1980s. In recent years, the advent of the biphasic waveform, better understanding of optimal shock waveform/duration, and higher-shock energy devices, have led some to question the need for defibrillation testing (DFT) [1]. Furthermore, controversy over whether to perform DFT has focused on possible adverse clinical events [1-4]. A prior systematic re- view by Phan et al. [5] demonstrates no significant benefit for DFT in terms of mortality, ICD efficacy or 30-day post-implant complications. In this systematic review and meta-analysis, we sought to update the effect of DFT on the risks of all-cause mortality, arrhythmic death, appropriate shock efficacy, and procedural adverse events.

\section{METHODS}

This analysis was performed in adherence to the Preferred 
Reporting Items for Systemic reviews and Meta-Analyses (PRISMA) statement on the quality of reporting of meta-analyses [6]ZirK'p.

\section{Search Strategy}

We searched the Medline (via PubMed), ClinicalTrials.gov, and the Cochrane Central Register of Controlled Trials for studies of DFT testing in ICD that had been published through June 31, 2017. Medical Subject Headings (MeSH) and keywords included the following: (1) implantable cardioverter-defibrillators, (2) DF test, (3) intra-operative DF testing, (4) ventricular tachycardia, and (5) ventricular fibrillation. In addition, we searched for meeting abstracts in Embase and hand-searched references and related citations in review articles and commentaries.

\section{Study Selection and Eligibility Criteria}

The primary outcome was all-cause mortality. Secondary outcomes included arrhythmic death and appropriate shock efficacy. Data on safety included procedural adverse events and complications as defined by the individual studies included. Only randomized studies that followed patients for $\geq 6$ months and in which mortality data were reported or available from the authors were included.

\section{Quality Assessment}

The internal validity of included studies was assessed using the Cochrane Collaboration's tool for assessing risk of bias in randomized trials [7].

\section{Data Synthesis and Statistical Analysis}

Data were pooled and analyzed by means of Review Manager (RevMan) [Computer program]. Version 5.3. Copenhagen: The Nordic Cochrane Centre, The Cochrane Collaboration, 2014. The random-effects model was used for the analyses. The effect size is presented as relative risk (RR). Statistical heterogeneity was evaluated using the $\mathrm{I}^{2}$ statistic and its $95 \%$ confidence interval (CI) [8]. A sensitivity analysis was performed by estimating the pooled effect sizes after leaving each study out one by one. A two-tailed $\mathrm{P} \leq 0.05$ was considered statistically significant.

\section{RESULTS}

\section{Study Selection}

Our literature search identified 484 publications (Fig 1). Sixteen articles were assessed for eligibility. Four RCTs met the inclusion criteria [Shockless IMPLant Evaluation (SIMPLE) [3], NO Regular Defibrillation testing In Cardioverter Defibrillator Implantation (NORDIC ICD) [9], Resynchronization for Ambulatory Heart Failure substudy (RAFT DFT) [10], and Test-No Test Implantable Cardioverter Defibrillator Pilot Study (TNT ICD) [11]]. These 4 studies included 3770 (1896 DFT and 1874 no-DFT) participants. Table 1 summarizes the characteristics and Fig 2 reported the quality assessment of the studies included.

\begin{tabular}{|c|c|c|c|}
\hline RAFT DFT & NORDIC ICD & SIMPLE & TNT ICD \\
\hline \multicolumn{4}{|l|}{ ClinicalTrials.gov Identifier } \\
\hline NCT00251251 & NCT01282918 & NCT00800384 & NCT01905007 \\
\hline \multicolumn{4}{|l|}{ Study design } \\
\hline Randomized 1:1 & Randomized 1:1 & Randomized 1:1 & Randomized 1:1 \\
\hline Paralell (2-arm) & Parallel (2-arm) & Parallel (2-arm) & Paralell (2-arm) \\
\hline Open-label & Open-label & Single-blind & Open-label \\
\hline Pilot & Non-inferiority & Non-inferiority & Pilot \\
\hline \multicolumn{4}{|l|}{ Centers } \\
\hline Multicenter, 34 sites & Multicenter, 48 sites & Multicenter, 85 sites & Multicenter, 2 sites \\
\hline \multicolumn{4}{|l|}{ Participants } \\
\hline 145 & 1077 & 2500 & 48 \\
\hline \multicolumn{4}{|l|}{ Inclusion criteria } \\
\hline $\begin{array}{l}\text { RAFT inclusion criteria (NYHA Class } \\
\text { II, LVEF } \leq 30 \% \text {; intrinsic QRS complex } \\
\text { width } \geq 120 \text { ms or paced QRS measure- } \\
\text { ment } \geq 200 \text { ms, ICD indication for pri- } \\
\text { mary or secondary prevention, optimal } \\
\text { heart failure pharmacological therapy, } \\
\text { normal sinus rhythm or chronic } \\
\text { persistent atrial tachyarrhythmia with } \\
\text { resting ventricular heart rate } \leq 60 \text { bpm } \\
\text { and } 6 \text { minute hall walk ventricular heart } \\
\text { rate of } \leq 90 \text { bpm; or chronic persistent } \\
\text { atrial tachyarrhythmia with resting } \\
\text { ventricular heart rate }>60 \text { bpm and } 6 \\
\text { minute hall walk ventricular heart rate }> \\
90 \text { bpm and booked for atrioventricular } \\
\text { junction ablation) }\end{array}$ & $\begin{array}{l}\text { Age } \geq 18 \text { years, initial ICD } \\
\text { implantation or CRT-D for Class } \\
\text { I indication according to the } \\
\text { ACC/AHA/ESC } 2006 \text { guideli- } \\
\text { nes and the } 2010 \text { focused update } \\
\text { of ESC guidelines on device } \\
\text { therapy in heart failure }\end{array}$ & $\begin{array}{c}\text { Age } \geq 18 \text { years, inizial implantation } \\
\text { of ICD or CRT-D }\end{array}$ & $\begin{array}{l}\text { Age } \geq 18 \text { years, } \\
\text { initial ICD implan- } \\
\text { tation or CRT-D for } \\
\text { Class I or Class II } \\
\text { indication according } \\
\text { to the ACC/AHA/ } \\
\text { HRS practice gui- } \\
\text { delines, anticipated } \\
\text { life expectancy }>6 \\
\text { months }\end{array}$ \\
\hline
\end{tabular}




\begin{tabular}{|c|c|c|c|}
\hline \multicolumn{4}{|l|}{ Exclusion criteria } \\
\hline $\begin{array}{l}\text { Documented intracardiac thrombus, } \\
\text { persistent/permanent AF without } \\
\text { appropriate anticoagulation, right-site } \\
\text { implant, inelegible for either DFT } \\
\text { strategy PLUS RAFT exlusion criteria } \\
\text { (intravenous inotropic agent in the last } \\
4 \text { days, life expectancy } \leq 1 \text { year from } \\
\text { non-cardiac cause, expected to undergo } \\
\text { cardiac transplantation within } 1 \text { year, } \\
\text { acute cardiac or non-cardiac illness that } \\
\text { requires intensive care, uncorrected or } \\
\text { uncorrectable primary valvular disease, } \\
\text { restrictive, hypertrophic, or reversible } \\
\text { form of cardiomyopathy, severe prima- } \\
\text { ry pulmonary hypertension, tricuspid } \\
\text { prosthetic valve, existing ICD, coronary } \\
\text { revascularization }<1 \text { month and } \\
\text { LVEF }>30 \% \text {, ACS with LVEF }>30 \% \text {, } \\
\text { included in another clinical trial that } \\
\text { will affect the objectives of this study, } \\
\text { history of noncompliance to medical } \\
\text { therapy) }\end{array}$ & $\begin{array}{l}\text { ARVC or hypertrophic cardiomy- } \\
\text { opathy, VF due to acute ischemia } \\
\text { or other potentially reversible } \\
\text { causes, actively listed for a trans- } \\
\text { plant, unable or unwilling to par- } \\
\text { ticipate in the study, unavailable } \\
\text { for required follow-ups and study } \\
\text { procedures, participating in an- } \\
\text { other clinical study other than a } \\
\text { registry or observational/non-in- } \\
\text { terventional study, anticipated } \\
\text { right sided implantation of ICD } \\
\text { generator, malignant condition } \\
\text { with a life expectancy less than } \\
\text { the duration of the study, preg- } \\
\text { nant and breast-feeding women, } \\
\text { terminal renal insufficiency, per- } \\
\text { sistent AF without pre-operative } \\
\text { TEE, persistent AF with left atrial } \\
\text { thrombus diagnosed by TEE }\end{array}$ & $\begin{array}{l}\text { Ineligible for either DFT strategy, } \\
\text { on active transplant list, unavail- } \\
\text { able for follow-up, pregnancy or } \\
\text { women of child bearing potential } \\
\text { not following an effective method } \\
\text { of contraception, anticipated right } \\
\text { sided implantation }\end{array}$ & $\begin{array}{l}\text { Contraindications } \\
\text { to DFT as de- } \\
\text { termined by the } \\
\text { managing physician, } \\
\text { ICD replacement } \\
\text { implants, right-sided } \\
\text { pectoral implants, } \\
\text { abdominal implants, } \\
\text { chronic oral } \\
\text { amiodarone therapy } \\
\text { (for }>6 \text { weeks and } \\
\text { continued need for } \\
\text { amiodarone), inabil- } \\
\text { ity to give informed } \\
\text { consent }\end{array}$ \\
\hline \multicolumn{4}{|l|}{ Device \& Sponsor } \\
\hline Medtronic & Biotronik & Boston Scientific & Medtronic \\
\hline \multicolumn{4}{|l|}{ Follow-up } \\
\hline 24.2 months (mean) & & 37 months (mean) & 14.9 months (mean) \\
\hline \multicolumn{4}{|l|}{ Primary endpoint } \\
\hline $\begin{array}{l}\text { SAFETY. Composite of death, stroke, } \\
\text { systemic embolism, myocardial } \\
\text { infarction, heart failure (requiring } \\
\text { intravenous diuretics, inotropes or } \\
\text { rehospitalization), hypotension (requir- } \\
\text { ing intravenous vaso- constrictors or } \\
\text { inotropes for > } 15 \text { minutes), need for } \\
\text { chest compressions or intraaortic bal- } \\
\text { loon pump, non-elective intubation or } \\
\text { aspiration, unplanned ICU stay, pneu- } \\
\text { mothorax, pericardial tamponade or } \\
\text { pericarditis, device infection (requiring } \\
\text { removal or IV antibiotics), arterial-line } \\
\text { complication (requiring intervention) } \\
\text { (within } 30 \text { days).EFFICACY. Com- } \\
\text { posite of failed appropriate ICD shock } \\
\text { or arrhythmic death }\end{array}$ & $\begin{array}{l}\text { Average efficacy of the first ICD } \\
\text { shock for all true ventricular } \\
\text { tachyarrhythmias }\end{array}$ & $\begin{array}{l}\text { Composite of failed approriate } \\
\text { shock or arrhythmic death }\end{array}$ & $\begin{array}{l}\text { Composite all-cause } \\
\text { mortality and oper- } \\
\text { ative complications } \\
\text { (within } 90 \text { days) }\end{array}$ \\
\hline \multicolumn{4}{|l|}{ Secondary endpoint } \\
\hline $\begin{array}{l}\text { All-cause mortality or hospitalization } \\
\text { for heart failure, all-cause mortality and } \\
\text { length of hospital stay }\end{array}$ & $\begin{array}{l}\text { SAFETY. Serious adverse events } \\
\text { (within } 30 \text { days), blood parame- } \\
\text { ters indicating myocardial injury } \\
\text { [BNP, Creatinine, Troponin T, } \\
\text { CK, CK-MB], system revisions } \\
\text { at implant, total fluoroscopy and } \\
\text { implantation time. EFFICACY. } \\
\text { All-cause mortality, cardiac } \\
\text { mortality, arrhythmic mortality, } \\
\text { ventricular tachyarrhythmia } \\
\text { conversion efficacy of the ICD } \\
\text { shock therapy }\end{array}$ & $\begin{array}{l}\text { Composite of death, stroke, } \\
\text { non-CNS systemic embolism, } \\
\text { pulmonary embolism, myocardial } \\
\text { infarction, heart failure (needing } \\
\text { inotropes or diuretics), intraop- } \\
\text { erative hypotension, need for } \\
\text { chest compression, non-elective } \\
\text { intubation, aspiration pneumonia, } \\
\text { unplanned ICU stay, pneumotorax, } \\
\text { pericarditis, cardiac perforation, par- } \\
\text { diac tamponade, device infection, } \\
\text { arterial-line complication, anoxic } \\
\text { brain injury (within } 30 \text { days) }\end{array}$ & $\begin{array}{c}\text { 1st shock efficacy for } \\
\text { clinical occurrence } \\
\text { of VT/VF }\end{array}$ \\
\hline \multicolumn{4}{|l|}{ Defibrillation testing } \\
\hline $1 / 1$ success at $15 \mathrm{~J}$ & $1 / 1$ success at $15 \mathrm{~J}$ & $1 / 1$ success at $17 \mathrm{~J}$ & $\mathrm{~N} / \mathrm{A}$ \\
\hline $1 / 1$ success at $25 \mathrm{~J}$ & $2 / 2$ success at $24 \mathrm{~J}$ & $2 / 2$ success at $21 \mathrm{~J}$ & $\mathrm{~N} / \mathrm{A}$ \\
\hline
\end{tabular}




\begin{tabular}{|c|c|c|c|}
\hline $\begin{array}{l}\text { VF-250 bpm; NID } 18 \text { of } 24 \text {; first VF } \\
\text { therapy 25J; maximum energy ( } \mathrm{J} \text { not } \\
\text { specified) x } 5\end{array}$ & $\begin{array}{l}\text { VF-222 bpm; NID } 12 \text { of } 16 \text {; ATP } \\
\text { one shot (if history of mono- } \\
\text { morphic VT in the zone of VF); } \\
40 \mathrm{~J} \text { x } 8\end{array}$ & $\begin{array}{l}\text { VF-230 bpm } / 250 \mathrm{bpm} ; 1 \mathrm{~s} \text { delay; } \\
\text { first VF therapy } 31 \mathrm{~J} \text {; second-last VF } \\
\text { terapy } 31 / 41 \mathrm{~J}\end{array}$ & N/A \\
\hline $\begin{array}{l}\text { FVT-200 bpm; NID } 18 \text { of } 24 \text {; ATP x } 1 \text {; } \\
\text { second FVT therapy } 25 \mathrm{~J} \text {; maximum } \\
\text { energy ( } \mathrm{J} \text { not specified) } \mathrm{x} 4\end{array}$ & $\begin{array}{l}\text { VT-188 bpm; NID 26; ATP at } \\
\text { discretion; 40 J x } 8\end{array}$ & $\begin{array}{l}\text { VT-180 bpm; } 2,5 \text { s delay; first VT } \\
\text { therapy: ATP x } 6 \text { (no in pts with } \\
\text { primary arrhythmic disorders); } \\
\text { second VT therapy } 31 \mathrm{~J} \text {; third-last } \\
\text { VT therapy } 31 / 41 \mathrm{~J}\end{array}$ & $\mathrm{~N} / \mathrm{A}$ \\
\hline
\end{tabular}

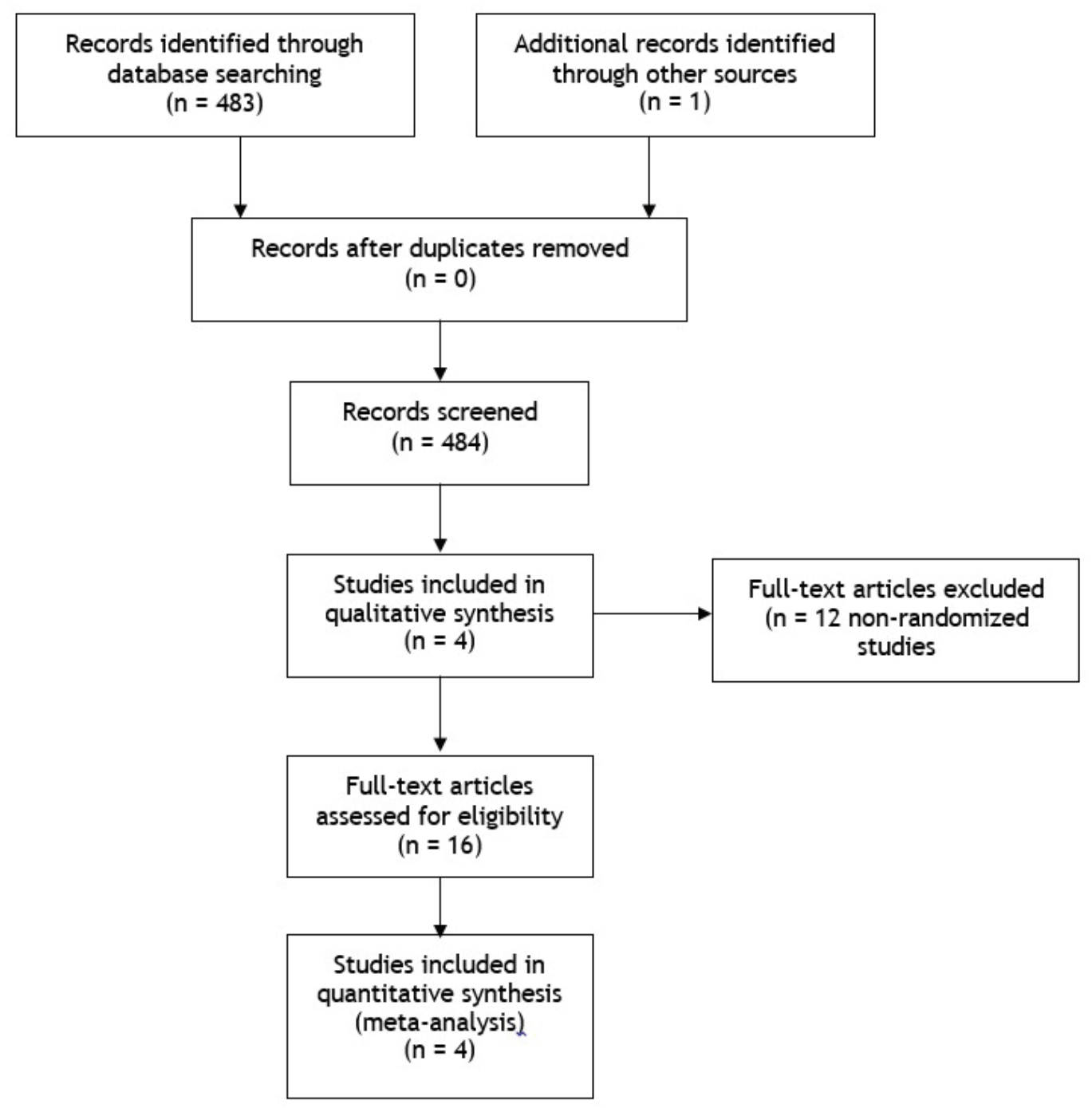

Figure 1: Flow Diagram of Eligible Studies 


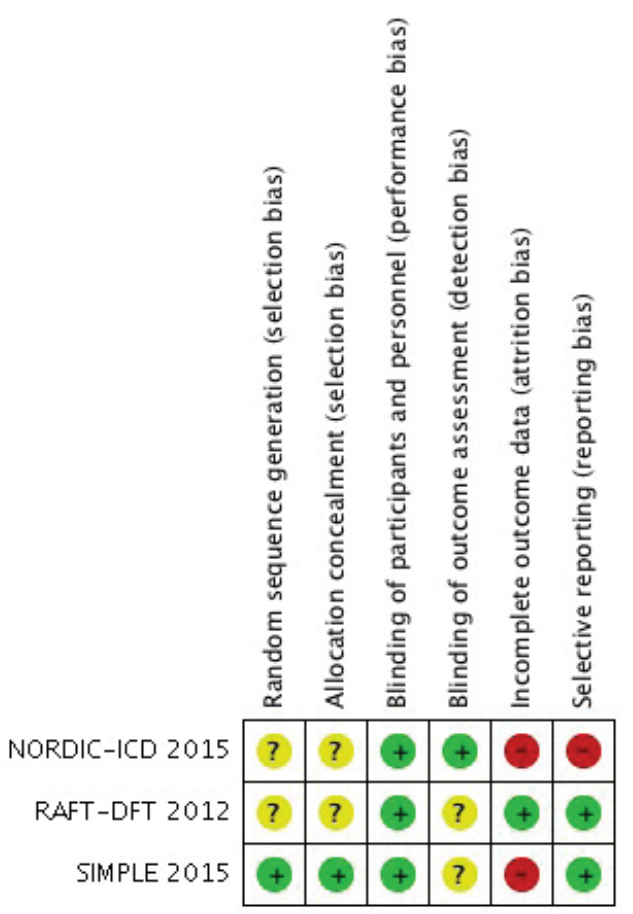

Figure 2: Risk of Bias Summary for the Studies Included. Green Indicates Low Risk of Bias. Red Indicates High Risk of Bias. Yellow Indicates Unclear Risk of Bias
RAFT DFT = Resynchronization/Defibrillation for Ambulatory Heart Failure Trial; DFT = defibrillation testing; NORDIC ICD = NO Regular Defibrillation Testing In Cardioverter Defibrillator Implantation (NORDIC ICD) Trial; SIMPLE = Shockless Implant Evaluation (SIMPLE) Trial; TNT ICD = TEST-NO TEST Implantable Cardioverter Defibrillator Pilot Study; ICD = implantable-cardioverter defibrillator; CRT-D = cardiac resynchronization therapy with ICD; ESC = European Society of Cardiology; ACC = American College of Cardiology; AHA = American Heart Association; HRS = Heart Rhythm Society; AF = atrial fibrillation; ARVC = arrhytmogrnic right ventricular cardiomyopathy; TEE = trans-esophgeal echocardiography; ICU = intensive care unit; $\mathrm{BNP}=\mathrm{B}$-type natriuretic peptide; $\mathrm{CK}=$ creatin kinase; $\mathrm{CK}-\mathrm{MB}=$ creatin kinase-MB; NYHA = New York heart Association; LVEF = left ventricular ejection fraction; ACS = acute coronary syndrome; IV = intravenous; $\mathrm{VT}=$ ventricular tachycardia; $\mathrm{VF}=$ ventricular fibrillation; $\mathrm{FVT}=$ fast ventricular tachycardia; $\mathrm{CNS}=$ central nervous system; $\mathrm{J}=$ Joules; NID = number of intervals to detect; $\mathrm{ATP}=$ antitachycardia pacing therapy; bpm, beats per minute; $\mathrm{s}=$ seconds; $\mathrm{N} / \mathrm{A}=$ not available.

\section{Characteristics}

The baseline characteristics of the patients are summarized in Table 2.

\begin{tabular}{|c|c|c|c|c|c|c|c|c|}
\hline & RAFT & DFT & NORDI & IC ICD & SIMI & PLE & TN' & ICD \\
\hline & $\operatorname{DFT}(+)$ & DFT (-) & $\operatorname{DFT}(+)$ & DFT (-) & $\operatorname{DFT}(+)$ & DFT (-) & $\operatorname{DFT}(+)$ & DFT (-) \\
\hline Population, $\mathrm{n}$ & 75 & 70 & 540 & 537 & 1253 & 1247 & 28 & 20 \\
\hline Age, years (SD) & $65.9(9.39)$ & $67.9(8.9)$ & $64.9(10.6)$ & $64.7(11.2)$ & $63.0(11.7)$ & $62.6(11.5)$ & $64.0(7.6)$ & $63.1(11.8)$ \\
\hline Male sex, n (\%) & $60(80)$ & $54(77)$ & $443(82.0)$ & $430(80.0)$ & $1009(80.5)$ & $1015(81.4)$ & $20(71.4)$ & $12(60.0)$ \\
\hline Ischemic disease & $\mathrm{N} / \mathrm{R}$ & $\mathrm{N} / \mathrm{R}$ & $360(66.7)$ & $341(63.5)$ & $799(63.8)$ & $821(65.8)$ & $16(59.3)$ & $11(55.0)$ \\
\hline $\begin{array}{l}\text { Non-ischemic dilated cardiomyop- } \\
\text { athy }\end{array}$ & $\mathrm{N} / \mathrm{R}$ & $\mathrm{N} / \mathrm{R}$ & $\mathrm{N} / \mathrm{R}$ & $\mathrm{N} / \mathrm{R}$ & $414(33.0)$ & $392(31.4)$ & $\mathrm{N} / \mathrm{R}$ & $\mathrm{N} / \mathrm{R}$ \\
\hline Hypertrophic cardiomyopathy & $\mathrm{N} / \mathrm{R}$ & $\mathrm{N} / \mathrm{R}$ & $\mathrm{N} / \mathrm{R}$ & $\mathrm{N} / \mathrm{R}$ & $53(4.2)$ & $42(3.4)$ & $\mathrm{N} / \mathrm{R}$ & $\mathrm{N} / \mathrm{R}$ \\
\hline $\begin{array}{l}\text { Long QT, Brugada syndrome, or } \\
\text { CPVT }\end{array}$ & $\mathrm{N} / \mathrm{R}$ & $\mathrm{N} / \mathrm{R}$ & $\mathrm{N} / \mathrm{R}$ & $\mathrm{N} / \mathrm{R}$ & $29(2.3)$ & $24(1.9)$ & $\mathrm{N} / \mathrm{R}$ & $\mathrm{N} / \mathrm{R}$ \\
\hline NYHA $\leq \mathrm{II}, \mathrm{n}(\%)$ & $75(100)$ & $70(100)$ & $266(49.3)$ & $257(47.9)$ & $410(32.7)$ & $404(32.4)$ & $\mathrm{N} / \mathrm{R}$ & $\mathrm{N} / \mathrm{R}$ \\
\hline NYHA $\geq$ III, n (\%) & $0(0)$ & $0(0)$ & $242(44.8)$ & $259(48.2)$ & $387(30.9)$ & $365(29.3)$ & $\mathrm{N} / \mathrm{R}$ & $\mathrm{N} / \mathrm{R}$ \\
\hline LVEF, \% (SD) & $24.7(4.6)$ & $23.6(4.6)$ & $\mathrm{N} / \mathrm{R}$ & $\mathrm{N} / \mathrm{R}$ & $32.0(12.8)$ & $31.6(12.4)$ & $\begin{array}{c}25.3 \\
(\mathrm{~N} / \mathrm{R})\end{array}$ & $\begin{array}{c}32.2 \\
(\mathrm{~N} / \mathrm{R})\end{array}$ \\
\hline Atrial fibrillation $\mathrm{n}(\%)$ & $4(5)$ & $4(6)$ & $40(7.4)$ & $45(8.4)$ & $139(11.1)$ & $141(11.3)$ & $\mathrm{N} / \mathrm{R}$ & $\mathrm{N} / \mathrm{R}$ \\
\hline Successful ICD implant n (\%) & $72 / 75(96)$ & $\begin{array}{l}70 / 70 \\
(100)\end{array}$ & $534(98.9)$ & $533(99.2)$ & $1242(99.1)$ & $1236(99.1)$ & $\mathrm{N} / \mathrm{R}$ & $\mathrm{N} / \mathrm{R}$ \\
\hline Patients received DFT, $\mathrm{n}(\%)^{\wedge}$ & $72(96)$ & $0(0)$ & $520(97.4)$ & $7(1.3)$ & $1218(98.1)$ & $9(0.7)$ & $\mathrm{N} / \mathrm{R}$ & $\mathrm{N} / \mathrm{R}$ \\
\hline $\begin{array}{l}\text { Intra-procedural system revision and } \\
\text { ICD re-programming } \mathrm{n} / \text { total (\%) }\end{array}$ & $3 / 71(4)$ & $0 / 0(0)$ & $\begin{array}{c}25 / 520 \\
(4.8)\end{array}$ & $0 / 7(0)$ & $\begin{array}{c}37 / 1119 \\
(3.3)\end{array}$ & $\mathrm{N} / \mathrm{R}$ & $\mathrm{N} / \mathrm{R}$ & $\mathrm{N} / \mathrm{R}$ \\
\hline Primary prevention, $\mathrm{n}(\%)$ & $71(94.7)$ & $66(94.3)$ & $439(81.3)$ & $434(81.0)$ & $924(73.7)$ & $889(71.3)$ & $23(82.1)$ & $20(100.0)$ \\
\hline Single-chamber ICD & $\mathrm{N} / \mathrm{R}$ & $\mathrm{N} / \mathrm{R}$ & $230(42.6)$ & $236(43.9)$ & $552(44.1)$ & $569(45.6)$ & $\mathrm{N} / \mathrm{R}$ & $\mathrm{N} / \mathrm{R}$ \\
\hline Dual-chamber ICD & $\mathrm{N} / \mathrm{R}$ & $\mathrm{N} / \mathrm{R}$ & $129(23.9)$ & $116(21.6)$ & $324(25.9)$ & $319(25,6)$ & $\mathrm{N} / \mathrm{R}$ & $\mathrm{N} / \mathrm{R}$ \\
\hline CRT-D, n (\%) & $37(49.3)$ & $39(55.7)$ & $175(32.4)$ & $181(33.6)$ & $366(29.2)$ & $348(27.9)$ & $\mathrm{N} / \mathrm{R}$ & $\mathrm{N} / \mathrm{R}$ \\
\hline Right-sided device implant & $\mathrm{N} / \mathrm{R}$ & $\mathrm{N} / \mathrm{R}$ & $3(0.5)$ & $6(1.1)$ & $13(1.0)$ & $15(1.2)$ & $\mathrm{N} / \mathrm{R}$ & $\mathrm{N} / \mathrm{R}$ \\
\hline Single-coil ICD lead & $\mathrm{N} / \mathrm{R}$ & $\mathrm{N} / \mathrm{R}$ & $254(47.6)$ & $262(49.2)$ & $\mathrm{N} / \mathrm{R}$ & $\mathrm{N} / \mathrm{R}$ & $\mathrm{N} / \mathrm{R}$ & $\mathrm{N} / \mathrm{R}$ \\
\hline Dual-coil ICD lead & $\mathrm{N} / \mathrm{R}$ & $\mathrm{N} / \mathrm{R}$ & $280(51.6)$ & $271(50.5)$ & $717(57.2)$ & $733(58.8)$ & $\mathrm{N} / \mathrm{R}$ & $\mathrm{N} / \mathrm{R}$ \\
\hline Amiodarone use, n (\%) & $12(16 \%)$ & $7(10)$ & $55(10.2)$ & $61(11.4)$ & $190(15.2)$ & $182(14.6)$ & $\mathrm{N} / \mathrm{R}$ & $\mathrm{N} / \mathrm{R}$ \\
\hline Beta-blockers use, n (\%) & $66(88)$ & $63(90)$ & $507(93.9)$ & $500(93.1)$ & $1088(86.8)$ & $1100(88.0)$ & $\mathrm{N} / \mathrm{R}$ & $\mathrm{N} / \mathrm{R}$ \\
\hline
\end{tabular}


RAFT-DFT = Resynchronization/Defibrillation for Ambulatory Heart Failure Trial; NORDIC-ICD = NO Regular Defibrillation Testing In Cardioverter Defibrillator Implantation Trial; SIMPLE = Shockless Implant Evaluation Trial; DFT = defibrillation testing; ICD = implantable cardioverter-defibrillator; CRT-D = ICD with resynchronization therapy; NYHA = New York Heart Association; LVEF = left ventricular ejection fraction; $\mathrm{CPVT}=$ catecholaminergic polymorphic ventricular tachycardia; DFT $(+)$ = defibrillation testing; DFT $(-)=$ no-defibrillation testing; N/R = not reported (One Patient in RAFTDFT and One Patient in NORDIC-ICD were not Inducible).

\section{Primary Outcome}

\section{All-Cause Mortality}

Data on mortality were analyzed in the intention-to-treat cohort. A total of 535 deaths (14.2\%) were reported. No significant difference in the risk of death $(R R=1.00,95 \%$ CI 0.86-1.17; P = 0.98) was observed with DFT versus noDFT (Fig 3). There was no significant heterogeneity among the 4 studies $\left(\mathrm{I}^{2}=0 \% ; \mathrm{P}=0.39\right)$. A sensitivity analysis revealed that no individual study had a predominant impact. However, the overall estimated RR increased to 1.24 (95\% CI 0.86-1.79; P = 0.24) when SIMPLE was excluded.

\section{Secondary Outcomes}

TNT ICD did not report the number of patients with ar- rhythmic death, and the number of first or any appropriate shock efficacy; so, the study was removed from the analyses.

\section{Arrhythmic Death}

Arrhythmic death occurred in 144 patients (3.9\%) and was similar between groups $(\mathrm{RR}=1.60,95 \% \mathrm{CI} 0.46-5.59$ : $\mathrm{P}=0.46)$ (Fig 4). There was no significant heterogeneity among the included studies $\left(\mathrm{I}^{2}=45 \% ; \mathrm{P}=0.18\right)$. On sensitivity analysis, while no individual study had a predominant impact, the overall estimated RR increased to 4.97 (95\% CI 0.58-42.42; P = 0.14) when SIMPLE was excluded.

\section{Shock Efficacy}

The analyses yielded the pooled effect-estimate in the on-treatment cohort. During follow-up, appropriate shock efficacy was reduced in the DFT group compared with the no-DFT group, with a statistically significant difference $(\mathrm{RR}=0.97,95 \%$ CI 0.95-1.00; $\mathrm{P}=0.02)$ (Fig 5). When we considered the first appropriate ICD shock, the pooled effect-estimate indicated a somewhat lower efficacy in the no-DFT group $(\mathrm{RR}=0.94,95 \% \mathrm{CI} 0.89-0.98 ; \mathrm{P}=0.004)$ (Fig 6). The sensitivity analysis suggested that NORDIC ICD had the greatest impact on statistical significance, and the overall estimated RR decreased to 0.96 (95\% CI 0.90$1.02 ; \mathrm{P}=0.17)$ when the study was omitted.

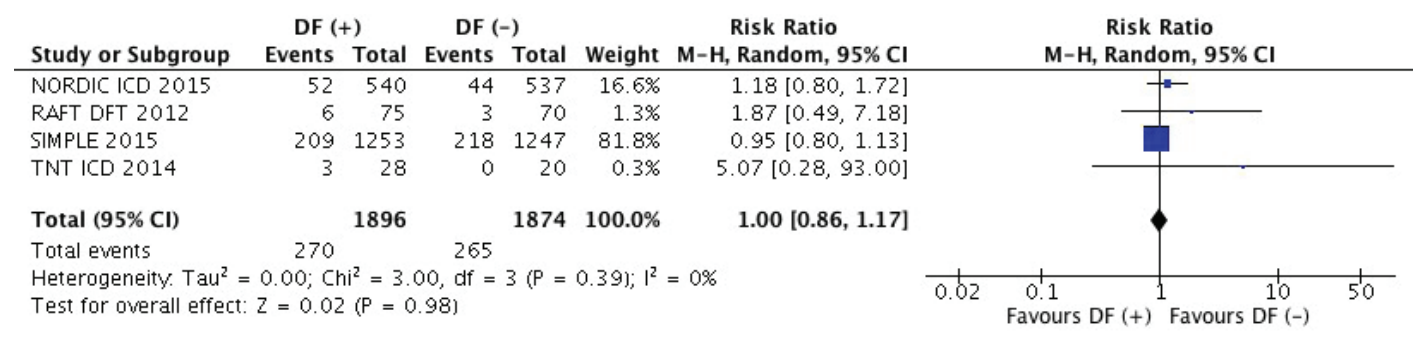

Figure 3: Meta-Analysis of All-Cause Mortality. The Risk Ratio for Mortality with DF (+) was not Statistically Significant from DF (-) Follow-Up. DF, Defibrillation Threshold. CI, Confidence Interval. M-H, Mantel-Haenszel.

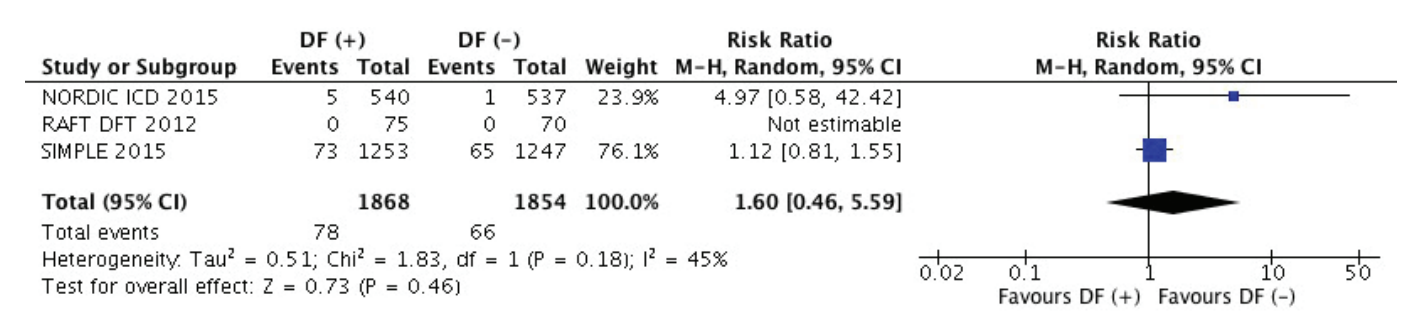

Figure 4: Meta-Analysis of Arrhythmic Death. The Risk Ratio for Arrhythmic Death with DF (+) was not Statistically Significant from DF (-) Follow-Up. Abbreviations as in Figure 3.

\begin{tabular}{|c|c|c|c|c|c|c|c|c|}
\hline Study or Subgroup & \multicolumn{2}{|c|}{ DF $(+)$} & \multicolumn{2}{|c|}{ DF (-) } & Weight & Risk Ratio & \multicolumn{2}{|c|}{$\begin{array}{c}\text { Risk Ratio } \\
\text { M-H, Random, } 95 \% \mathrm{CI}\end{array}$} \\
\hline NORDIC ICD 2015 & 204 & 211 & 218 & 218 & $71.0 \%$ & $0.97[0.94,0.99]$ & -1 & \\
\hline RAFT DFT 2012 & 16 & 16 & 19 & 19 & $4.2 \%$ & $1.00[0.90,1.11]$ & & \\
\hline SIMPLE 2015 & 181 & 191 & 180 & 188 & $24.8 \%$ & $0.99[0.95,1.04]$ & $\rightarrow$ & - \\
\hline Total $(95 \% \mathrm{Cl})$ & & 418 & & 425 & $100.0 \%$ & $0.97[0.95,1.00]$ & & \\
\hline Total events & 401 & & 417 & & & & & \\
\hline $\begin{array}{l}\text { Heterogeneity. } \mathrm{Tau}^{2}= \\
\text { Test for overall effect: }\end{array}$ & $\begin{array}{l}0.00 ; \mathrm{Cr} \\
\mathrm{z}=2.32\end{array}$ & $\begin{array}{l}i^{2}=1 . \\
(P=0\end{array}$ & $\begin{array}{l}17, \mathrm{df}= \\
.02 \mathrm{~J}\end{array}$ & $2(P=$ & $0.56 \mathrm{j} ;$ & $=0 \%$ & $\begin{array}{c}0.85 \quad 0.9 \\
\text { Fewer efficacy in DF (+) }\end{array}$ & $\begin{array}{cc}1.1 & 1.2 \\
\text { Fewer efficacy in } \mathrm{DF}(-)\end{array}$ \\
\hline
\end{tabular}

Figure 5: Meta-Analysis of any Appropriate Shock Efficacy. DF (+) Demonstrated A Nonsignificant Trend toward Fewer Appropriate Shock Efficacy. Abbreviations as in Figure 3. 


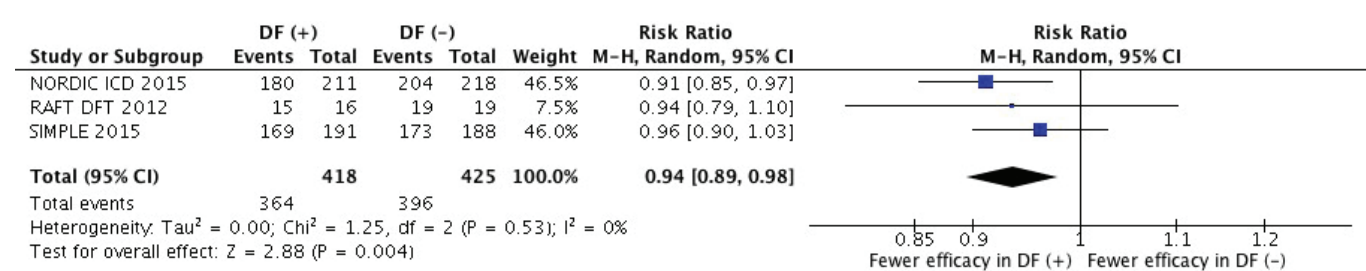

Figure 6: Meta-Analysis of First Appropriate Shock Efficacy. DF (+) Demonstrated A Significant Fewer First Appropriate Shock Efficacy. Abbreviations as in Figure 3.

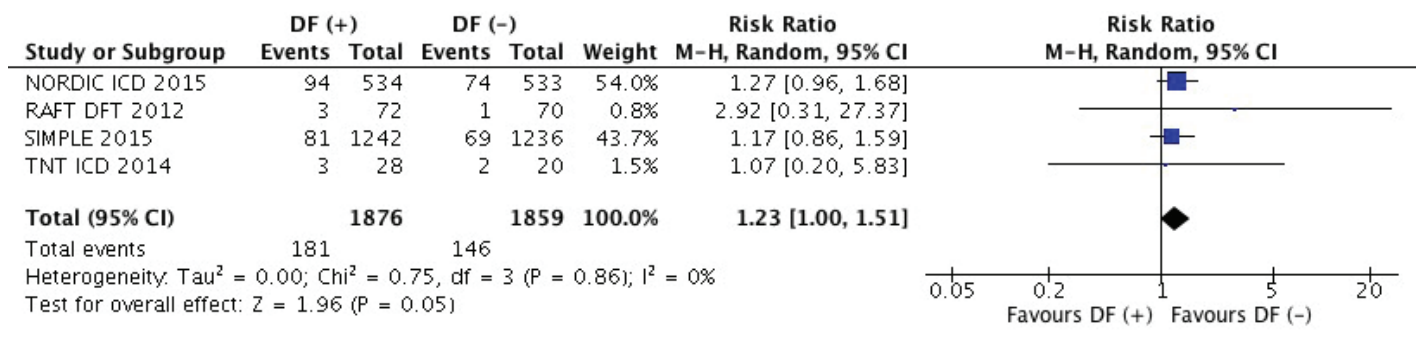

Figure 7: Meta-Analysis of Major Adverse Events. Pooled Data Showed A Significant Reduction in the Risk of Adverse Events with DF (-). Abbreviations as in Figure 3.

\section{Safety Outcomes}

A total of 327 patients had procedural adverse events and complications related to ICD implantation, 181 of whom had undergone DFT and 146 had not $(\mathrm{RR}=1.23$; $95 \% \mathrm{CI}$ $1.00-1.51 ; \mathrm{P}=0.05)$ (Fig 7). There was no statistical heterogeneity among the studies $\left(\mathrm{I}^{2}=0 \% ; \mathrm{P}=0.86\right)$. On sensitivity analysis, two studies had a major impact on the statistical analysis; the overall estimated RR decreased to 1.18 (95\% CI $0.87-1.60 ; \mathrm{P}=0.27$ ) when NORDIC ICD was excluded, and increased to 1.28 (95\% CI 0.97-1.68; P = 0.08) when SIMPLE was omitted. The 30-day procedure-related mortality rate was $0.3 \%$ in the DFT group and $0.5 \%$ in the no-DFT group $(\mathrm{RR}=0.69,95 \% \mathrm{CI} 0.25-1.88 ; \mathrm{P}=0.46)$. The procedure-related stroke rate was $0.2 \%$ in patients with DFT and $0.4 \%$ in those without DFT $(\mathrm{RR}=0.61,95 \%$ CI $0.17-2.25$; $\mathrm{P}=0.46$ ). Finally, intraoperative hypotension was the only adverse event somewhat more frequent in patients with DFT (1.1\% vs. $0.3 \%$; RR = 3.83, 95\% CI $0.69-21.31$; $\mathrm{P}=0.13$ ).

\section{DISCUSSION}

\section{Findings}

The findings from our systematic review and meta-analysis suggest that DFT at the time of ICD implantation has no impact on all-cause mortality or arrhythmic death during follow-up. Unexpectedly, the effect estimate reveals a $2 \%$ to $11 \%$ statistically significant lower first appropriate shock efficacy in the group with DFT versus those with no-DFT. This outcome was greatly influenced by the results of NORDIC ICD, which used higher programmed first shock energy (40 J) than SIMPLE (31J) and RAFT DFT (25J). Furthermore, a reduction was found in any appropriate shock efficacy in interrupting ventricular arrhythmias in the DFT group. This result was not affected by any single study, as the shocks delivered after the first were programmed to the maximum energy (from $31 \mathrm{~J}$ to $41 \mathrm{~J}$ ) and did not differ greatly in number in the included studies. RAFT DFT, SIMPLE, NORDIC ICD, and TNT ICD demonstrated similar modest increases in overall procedural safety outcomes, which did not quite reach statistical significance in any individual trial. When we pooled the data, DFT was associated with a statistically significant $23 \%$ increased risk of total procedural adverse events and complications. This result was mainly driven by a single adverse event (intraoperative hypotension).

\section{Strengths of our Analysis}

To the best of our knowledge, this is the first systematic review and meta-analysis of RCTs to compare the efficacy and safety of DFT at time of ICD implantation. Our results are consistent with previous observational data [12-22] and meta-analyses $[5,23]$ and provide further support to the recommendation to omit defibrillation efficacy testing in patients undergoing initial transvenous ICD implantation procedures [24]. Nonetheless, these results do not apply to specific subgroups, which were excluded or poorly represented in this meta-analysis, such as patients with right-sided ICD pocket or non-transvenous systems; patients with congenital heart diseases, channelopathies or hypertrophic cardiomyopathy; and patients who undergo device replacement. Ventricular fibrillation is induced during ICD implantation to assess: (1) electrical integrity of the connections between the leads and pulse generator; (2) reliable sensing, detection, and redetection in VF; and (3) optimal, or at least adequate, programmed shock strength. Low voltage pulses or shocks in normal rhythm can achieve the first goal. Concerning the second point, several studies have demonstrated a strong correlation between $\mathrm{R}$ wave amplitude in native rhythm and reliable sensing during induced and spontaneous VF. If the $\mathrm{R}$ wave during native rhythm is $\geq 5-7 \mathrm{mV}$, sensing during VF is almost always sufficient to ensure rapid detection or redetection $[25,26]$. Based on these considerations, the issue of 
whether to perform DFT is confined to the third point. Assessing the value of DFT requires considering four questions: (1) Does it predict shock success for induced VF? (2) Does it predict shock success for spontaneous VT/VF? (3) What is the relationship between DFT and conversion of spontaneous ventricular tachyarrhythmias? (4) Does it predict total mortality or sudden death? The studies included in our meta-analysis used a $10 \mathrm{~J}$ "safety-margin" criterion on implantation. This method limits testing to the minimum number of induced episodes necessary to determine whether there is a sufficient safety-margin (i.e. $10 \mathrm{~J}$ ) between the maximum shock strength of the ICD and the shock strength required for consistent defibrillation. Data from the studies indicate that an extremely high number of patients (97.1\%) were successfully defibrillated at the tested shock strength. While device revision provided no benefit during follow-up, patients in the DFT group underwent unnecessary system revision and/or ICD reprogramming [27]. The primary assumption of intraoperative DFT is that successful defibrillation on implantation will predict successful treatment of clinical ventricular arrhythmias. Our results indicate that the efficacy of the firstshock in interrupting spontaneous rapid VT or VF ranged from $87.0 \%$ in the DFT patients to $93.2 \%$ in the no-DFT group. This observation suggests that shocks for spontaneous VT/VF may not be effective for reasons that are not evaluated on implantation, therefore negating the utility of DFT. Furthermore, the first-shock success rate in spontaneous $\mathrm{VT} / \mathrm{VF}$ has a weak relationship with the total conversion rate for VT/VF. Implantable cardioverter-defibrillators deliver up to six or eight shocks for VT/VF at maximum strength, so that subsequent shocks may succeed if the first fails, and better implant testing did not reduce overall shock efficacy. Finally, DFT had a neutral effect on mortality. Data regarding the relationship between ICD implant testing and either total mortality or arrhythmic death are limited and difficult to interpret for several reasons. Factors that cannot be tested on implantation probably cause some failed shocks or sudden death. Such factors include ischemia, progressive heart failure, metabolic abnormalities, drug effects, and ICD lead or generator failures. Further, it is difficult to establish how often a high DFT is caused by an inadequate ICD system, and how often it is an indirect marker of a "sicker" patient. It is reasonable to accept that patients with unreliable defibrillation on implantation have a clinically higher risk of sudden death. Finally, some sudden cardiac deaths in ICD patients are caused by malfunctions of ICD leads or pulse generators, which are undetectable at time of implantation $[28,29]$. Another important question for this review concerns the risks of defibrillation on implantation. The risks of DFT include those related to induction of VF and those related to shocks alone. The anesthesia required for the delivery of shocks is another potential cause of complications. Data from our meta-analysis indicate that adverse events and complications following ICD implantation are uncommon, and rarely lead to death or permanent disability. Therefore, specific complications that occurred more frequently in patients with DFT were typically short-lived, such as intraoperative hypotension.

\section{Limitations}

The results of our meta-analysis are weakened by limitations inherent in meta-analyses and in the included studies. The low number of studies included meant that statistical power was low, especially for safety data analysis. The analysis of rare events carries its own limitations, in that even a small change in the number of events can produce a dramatic change in the results. Our pooled effect estimate included trials that differed in terms of follow-up. NORDIC ICD had a shorter follow-up than SIMPLE (22.8 versus 37 months) and, although the patient populations in the two studies were comparable, the overall mortality rate was lower in NORDIC ICD, which is indicative of a "less sick" patient cohort. RAFT DFT had a follow-up comparable with that of NORDID ICD, but included only patients in NYHA II functional class. It is noteworthy that NORDIC ICD collected data not only on protocol-specified complications but also on all procedureand patient-related adverse events, which may have affected the results of our safety analysis. Finally, there was a lack of detailed information on intra-operative complications in NORDIC ICD and TNT ICD. SIMPLE and NORDIC ICD reported losses to follow-up that were greater in each trial's no-DFT group, though the overall difference of being lost to follow-up was not statistically significant $(\mathrm{RR}=1.62 ; 95 \% \mathrm{CI}$ $0.90-2.93 ; \mathrm{P}=0.11$ ).

This meta-analysis demonstrated that routine DFT at the time of ICD implantation was substantially safe, but did not improve shock efficacy or reduce mortality in comparison with the no-testing strategy. It is therefore expected that the results of the meta-analysis will lead to abandonment of the practice of VF testing in selected patients who underwent transvenous ICD implantation.

\section{REFERENCES}

1. Viskin S, Rosso R. The top 10 reasons to avoid defibrillation threshold testing during ICD implantation. Heart Rhythm. 2008;5(3):3913. DOI: $10.1016 /$ j.hrthm.2008.01.006 PMID: 18313596

2. Birnie D, Tung S, Simpson C, Crystal E, Exner D, Ayala Paredes FA, et al. Complications associated with defibrillation threshold testing: the Canadian experience. Heart Rhythm. 2008;5(3):387-90. DOI: 10.1016/j.hrthm.2007.11.018 PMID: 18243813

3. Healey JS, Hohnloser SH, Glikson M, Neuzner J, Mabo P, Vinolas $\mathrm{X}$, et al. Cardioverter defibrillator implantation without induction of ventricular fibrillation: a single-blind, non-inferiority, randomised controlled trial (SIMPLE). Lancet. 2015;385(9970):785-91. DOI: 10.1016/S0140-6736(14)61903-6 PMID: 25715991

4. Semmler V, Biermann J, Haller B, Jilek C, Sarafoff N, Lennerz C, et al. ICD Shock, Not Ventricular Fibrillation, Causes Elevation of High Sensitive Troponin T after Defibrillation Threshold Testing-The Prospective, Randomized, Multicentre TropShock-Trial. PLoS One. 2015;10(7):e0131570. DOI: 10.1371/journal.pone.0131570 PMID: 26208329

5. Phan K, Ha H, Kabunga P, Kilborn MJ, Toal E, Sy RW. Systematic Review of Defibrillation Threshold Testing at De Novo Implantation. Circ Arrhythm Electrophysiol. 2016;9(4):e003357. DOI: 10.1161/CIRCEP.115.003357 PMID: 27030699

6. Liberati A, Altman DG, Tetzlaff J, Mulrow C, Gotzsche PC, Ioannidis JP, et al. The PRISMA statement for reporting systematic reviews and meta-analyses of studies that evaluate healthcare interventions: explanation and elaboration. BMJ. 2009;339:b2700. DOI: 10.1136/ bmj.b2700 PMID: 19622552

7. Higgins JP, Green S. Cochrane Handbook for Systematic Reviews of Interventions: John Wiley \& Sons; 2014. 154-5 p

8. Higgins JP, Thompson SG, Deeks JJ, Altman DG. Measuring inconsistency in meta-analyses. BMJ. 2003;327(7414):557-60. DOI: 10.1136/bmj.327.7414.557 PMID: 12958120

9. Bansch D, Bonnemeier H, Brandt J, Bode F, Svendsen JH, Taborsky $\mathrm{M}$, et al. Intra-operative defibrillation testing and clinical shock efficacy in patients with implantable cardioverter-defibrillators: the NORDIC ICD randomized clinical trial. Eur Heart J. 2015;36(37):25007. DOI: $10.1093 /$ eurheartj/ehv292 PMID: 26112885 
10. Healey JS, Gula LJ, Birnie DH, Sterns L, Connolly SJ, Sapp J, et al. A randomized-controlled pilot study comparing ICD implantation with and without intraoperative defibrillation testing in patients with heart failure and severe left ventricular dysfunction: a substudy of the RAFT trial.J Cardiovasc Electrophysiol. 2012;23(12):1313-6. DOI: 10.1111/i.1540-8167.2012.02393.x PMID: 22788915

11. Russo A, Andriulli J, Ortman M. Outcome Following ICD Implantation With Versus Without Defibrillation Testing: Preliminary Results of The Prospective Randomized Test-No Test (TNT) Pilot Study. J Am Coll Cardiol. 2014;12(63):451-6.

12. Ashino S, Nakai T, Sonoda K, Sasaki N, Kurokawa S, Ikeya Y, et al. Assessment of Efficacy and Necessity of Routine Defibrillation Threshold Testing in Patients Undergoing Implantable Cardioverter-Defibrillator (ICD) Implantation. Int Heart J. 2015;56(6):61821. DOI: $10.1536 /$ ihj.15-093 PMID: 26549282

13. Hadid C, Atienza F, Strasberg B, Arenal A, Codner P, Gonzalez-Torrecilla $\mathrm{E}$, et al. Clinical impact of defibrillation testing at the time of ICD insertion. Cardiol J. 2014. DOI: 10.5603/CJ.a2014.0062 PMID: 25179316

14. Arnson Y, Suleiman M, Glikson M, Sela R, Geist M, Amit G, et al. Role of defibrillation threshold testing during implantable cardioverter-defibrillator placement: data from the Israeli ICD Registry. Heart Rhythm. 2014;11(5):814-21. DOI: 10.1016/j. hrthm.2014.01.030 PMID: 24486799

15. Brignole M, Occhetta E, Bongiorni MG, Proclemer A, Favale S, Iacopino S, et al. Clinical evaluation of defibrillation testing in an unselected population of 2,120 consecutive patients undergoing first implantable cardioverter-defibrillator implant. J Am Coll Cardiol. 2012;60(11):981-7. DOI: 10.1016/j.jacc.2012.05.014 PMID: $\underline{22858384}$

16. Michowitz Y, Lellouche N, Contractor T, Bourke T, Wiener I, Buch E, et al. Defibrillation threshold testing fails to show clinical benefit during long-term follow-up of patients undergoing cardiac resynchronization therapy defibrillator implantation. Europace. 2011;13(5):683-8. DOI: 10.1093/europace/euq519 PMID: 21252192

17. Calvi V, Dugo D, Capodanno D, Arancio R, Di Grazia A, Liotta C, et al. Intraoperative defibrillation threshold testing during implantable cardioverter-defibrillator insertion: do we really need it? Am Heart J. 2010;159(1):98-102. DOI: 10.1016/j.ahj.2009.10.031 PMID: 20102873

18. Bianchi S, Ricci RP, Biscione F, Sgreccia F, Di Belardino N, Rossi P, et al. Primary prevention implantation of cardioverter defibrillator without defibrillation threshold testing: 2-year follow-up. Pacing Clin Electrophysiol. 2009;32(5):573-8. DOI: 10.1111/j.15408159.2009.02329.x PMID: 19422577

19. Hall B, Jeevanantham V, Levine E, Daubert J, McNitt S, Hall F, et al. Comparison of outcomes in patients undergoing defibrillation threshold testing at the time of implantable cardioverter-defibrillator implantation versus no defibrillation threshold testing. Cardiol J. 2007;14(5):463-9. PMID: 18651506

20. Brignole M, Raciti G, Bongiorni MG, De Martino G, Favale S, Gasparini $\mathrm{M}$, et al. Defibrillation testing at the time of implantation of cardioverter defibrillator in the clinical practice: a nation-wide survey. Europace. 2007;9(7):540-3. DOI: 10.1093/europace/eum083 PMID: 17507358

21. Pires LA, Johnson KM. Intraoperative testing of the implantable cardioverter-defibrillator: how much is enough? J Cardiovasc Electrophysiol. 2006;17(2):140-5. DOI: 10.1111/j.15408167.2005.00294.x PMID: 16533250

22. Russo AM, Sauer W, Gerstenfeld EP, Hsia HH, Lin D, Cooper JM, et al. Defibrillation threshold testing: is it really necessary at the time of implantable cardioverter-defibrillator insertion? Heart Rhythm. 2005;2(5):456-61. DOI: 10.1016/j.hrthm.2005.01.015 PMID: 15840466

23. Stavrakis S, Patel NH, Reynolds DW. Defibrillation threshold testing does not predict clinical outcomes during long-term follow-up: a meta-analysis. Pacing Clin Electrophysiol. 2013;36(11):1402-8. DOI: $10.1111 /$ pace.12218 PMID: 23875736

24. Wilkoff BL, Fauchier L, Stiles MK, Morillo CA, Al-Khatib SM, Almendral J, et al. 2015 HRS/EHRA/APHRS/SOLAECE expert consensus statement on optimal implantable cardioverter-defibrillator programming and testing. Heart Rhythm. 2016;13(2):e50-86. DOI 10.1016/j.hrthm.2015.11.018 PMID: 26607062

25. Swerdlow CD. Implantation of cardioverter defibrillators without induction of ventricular fibrillation. Circulation. 2001;103(17):215964. PMID: 11331256

26. Ellenbogen KA, Wood MA, Stambler BS, Welch WJ, Damiano RJ Measurement of ventricular electrogram amplitude during intraoperative induction of ventricular tachyarrhythmias. Am J Cardiol. 1992;70(11):1017-22. PMID: 1414898

27. Degroot PJ, Church TR, Mehra R, Martinson MS, Schaber DE. Derivation of a defibrillator implant criterion based on probability of successful defibrillation. Pacing Clin Electrophysiol. 1997;20(8 Pt 1):1924-35. PMID: 9272530

28. Gornick CC, Hauser RG, Almquist AK, Maron BJ. Unpredictable implantable cardioverter-defibrillator pulse generator failure due to electrical overstress causing sudden death in a young high-risk patient with hypertrophic cardiomyopathy. Heart Rhythm. 2005;2(7):6813. DOI: 10.1016/i.hrthm.2005.05.014 PMID: 15992719

29. Hauser RG, Hayes DL, Almquist AK, Epstein AE, Parsonnet V, Tyers $\mathrm{GF}$, et al. Unexpected ICD pulse generator failure due to electronic circuit damage caused by electrical overstress. Pacing Clin Electrophysiol. 2001;24(7):1046-54. PMID: 11475818 